\title{
PHARMACOLOGICAL ASPECTS OF NEWER DRUGS USED IN ANTICOAGULANT THERAPY
}

\author{
Naveen Kumar.T, Naser Ashraf Tadvi, S. Mabu Shareef, Sateesh K. Malkappa
}

1. Associate Professor. Department of Pharmacology, Apollo institute of Medical Sciences and Research, Apollo health city campus, Jubilee hills , Hyderabad.

2. Associate Professor. Department of Pharmacology, Kamineni Institute of Medical Sciences. Sreepuram, Narketpally, Andhra Pradesh.

3. Assistant Professor. Department of Pharmacology, Kamineni Institute of Medical Sciences. Sreepuram, Narketpally, Andhra Pradesh.

4. Assistant Professor. Department of Microbiology, Kamineni Institute of Medical Sciences. Sreepuram, Narketpally, Andhra Pradesh.

\section{CORRESPONDING AUTHOR}

Dr. Naveen Kumar. T.

Associate Professor, Department of Pharmacology.

Apollo Institute of Medical Sciences and Research,

Apollo Health City Campus. Jubilee Hills,

Hyderabad, Andhra Pradesh - 508254

E-mail: doctornaveen1@rediffmail.com

Ph: 00919885227846.

ABSTRACT: ${ }^{1}$ Parenteral low molecular weight heparins like enoxaparin, dalteparin, tinzaparin, synthetic heparin derivatives fondaparinux, direct thrombin inhibitors like lepirudin, bivalirudin, argatroban, oral anticoagulants like Dabigatran, Rivaroxiban are newer anticoagulants. The emergence of new oral anticoagulants has the advantage over old drugs is their predictable pharmacokinetics, pharmacodynamics properties, adverse effects clinically less relevant and their different mechanism of action.

Clinically these new anticoagulant drugs are preferred for prophylaxis of patients undergoing hip or knee surgery to prevent deep vein thrombosis, after the replacement of heart valve, open- heart surgery to prevent clot formation and for therapy of pulmonary embolism (synthetic derivatives). Also as an alternative in patients diagnosed with heparin induced thrombocytopenia who require anticoagulation ${ }^{2}$.

KEY WORDS: LMWH, DTI, Dabigatran, Rivaroxiban

INTRODUCTION: Anticoagulants are drugs used to prevent clot formation or prevent clot enlargement by inhibiting the action of clotting factors or platelets. The limitations of existing oral and parenteral anticoagulants have prompted a search for novel agents. Focusing on new anticoagulants this topic discusses the mechanism of coagulation, describes the methods for testing new anticoagulant agents and Provides clinical perspective as to which these new strategies is most likely to succeed. 
Fig no 1 Coagulation pathway and sites of action of drugs

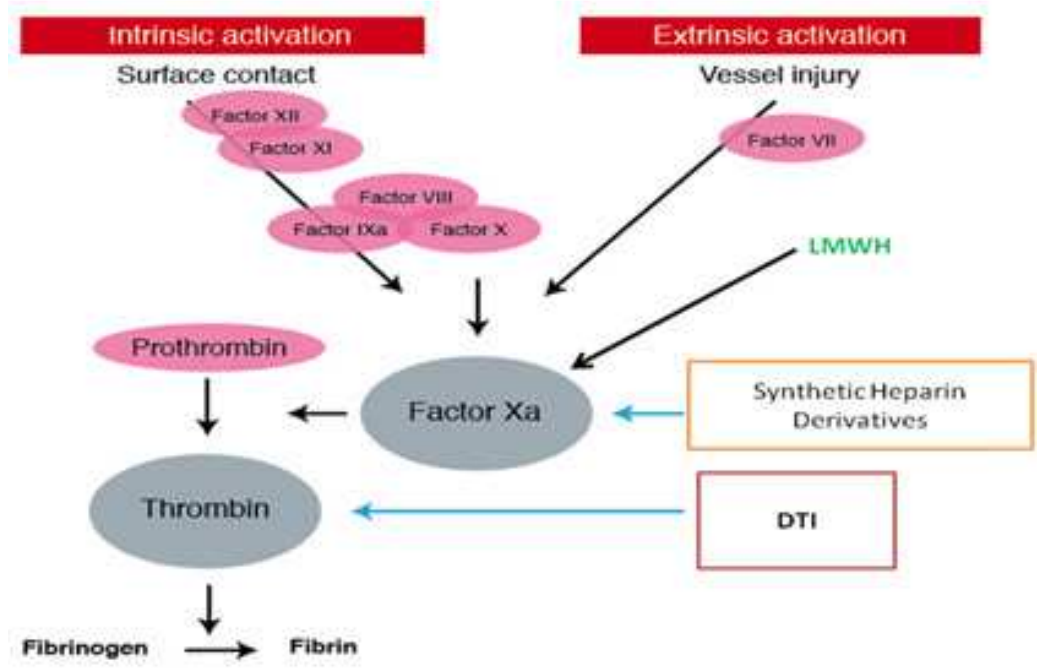

MECHANISM OF ACTION OF NEWER ANTICOAGULANTS: Thrombin-inhibiting drugs can block the action of thrombin by binding to three domains: the active site or catalytic site and two exosites. Located next to the active site, exosite 1 acts as a dock for substrates such as fibrin, there by orienting the appropriate peptide bonds in the active site. Exosite 2 serves as the heparin-binding domain. Thrombin is inhibited indirectly by low molecular weight heparins, because these drugs strongly catalyze the function of antithrombin. A heparin-thrombinantithrombin complex is formed in which heparin binds simultaneously to exosite 2 in thrombin and to antithrombin. Furthermore, heparin can act as a bridge between thrombin and fibrin by binding both to fibrin and exosite 2.Because both thrombin exosites are occupied within this fibrin-heparin-thrombin complex, the enzymatic activity of thrombin is relatively protected from inactivation by the heparin-antithrombin complex. Thus, heparins have a reduced capacity for the inhibition of fibrin-bound thrombin, which appears to be detrimental, because active thrombin further triggers thrombus growth.

Since DTIs act independently of antithrombin, they can inhibit thrombin bound to fibrin or fibrin degradation products. Bivalent DTIs block thrombin at both the active site and exosite 1 , whereas univalent DTIs bind only to the active site. The group of bivalent DTIs includes hirudin and bivalirudin, whereas argatroban and dabigatran are univalent DTIs. Native hirudin and recombinant hirudins (lepirudin and desirudin) form an irreversible 1:1 stoichiometric complex with thrombin. In a similar way, bivalirudin, a synthetic hirudin, binds to the active site and exosite 1, but once bound, it is cleaved by thrombin, thereby restoring the active-site functions of thrombin. Therefore, in contrast to the hirudins, bivalirudin produces only a transient inhibition of thrombin. In summary low molecular weight heparin (LMWH), Synthetic heparin derivatives inhibit factor Xa predominantly, has less effect on thrombin, platelet function and on coagulation. ${ }^{3}$ Direct thrombin inhibitors (DTIs) act by directly inhibiting thrombin to delay clotting. ${ }^{3}$

\section{PHARMACOLOGICAL ROLE OF NEW ORAL AND PARENTERAL DRUGS:}

(1) LMWH: Low molecular weight heparins are given by subcutaneous route. They have better bio-availability (70-90\%), variability in response is minimised. ${ }^{4}$ incidence of adverse effects like thrombocytopenia are less frequent and chance of haemorhage is less. These drugs have long 
elimination half life and are eliminated by first order kinetics, so effects are more consistent and dosing is less frequent (once daily). These do not prolong aPTT and whole blood clotting time. Dose given in milligram, can be easily calculated based on body weight

(2) Synthetic heparin derivatives: (fondaparinux): These drugs have less antiplatelet action and hence, chance of thrombocytopenia is less frequent. Given subcutaneously once daily (Both LMWH and synthetic derivatives are used for prophylaxis of patients undergoing hip or knee surgery, after the replacement of heart valve, open-heart surgery and for therapy of pulmonary embolism)

(3) Direct thrombin inhibitors (Parenteral): These drugs are especially useful in cases of heparin induced thrombocytopenia (HIT). Direct thrombin inhibitors are of 2 types: monovalent and bivalent. ${ }^{6}$

(a) The bivalent include bivalirudin and lepirudin; bind both to the active site and Exosite 1 (E1) of thrombin.

(b) Univalent DTIs bind only the active site and include Argatroban and Dabigatran

Fig No:2

A.

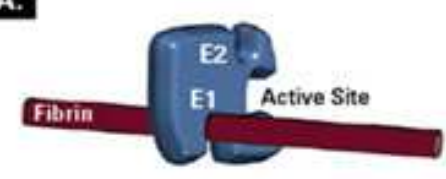

Fibrin-bound Thrombin

C.

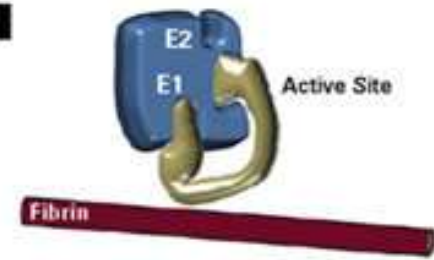

Bivalent: Lepirudin
B.

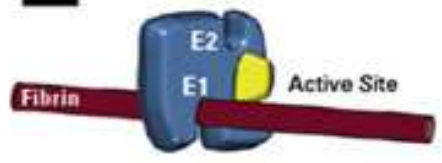

Univalent: Argatroban \& Dabigatran

D.

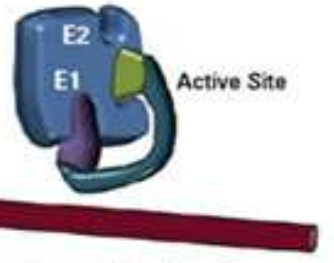

Bivalent: Bivalirudin

Table no 1: Pharmacokinetics parameters of DTI

\begin{tabular}{|l|l|l|l|l|}
\hline Parameter & Bivalirudin & Lepirudin & Argatroban & Dabigatran \\
\hline Class & Bivalent & Bivalent & Univalent & Univalent \\
\hline Indication & HIT & HIT & HIT & Sroke prevention \\
\hline Administration & Parenteral(IV) & Parenteral(IV) & Parenteral(IV) & Oral \\
\hline Plasmahalf life & $20-30$ min & $1 \mathrm{hr}$ & $45 \mathrm{~min}$ & $14-17 \mathrm{hrs}$ \\
\hline $\begin{array}{l}\text { Time of peak } \\
\text { Concentration }\end{array}$ & 0.5 to 1.5 hours & 2 to 3 hours & 2 to 4 hours & 1.5 to 2hours \\
\hline Clearance & Enzymatic & Kidneys & Liver & Kidneys \\
\hline Monitoring & aPTT & aPTT & aPTT & None \\
\hline
\end{tabular}

HIT - Heparin induced thrombocytopenia, aPTT- activated partial thromboplastin time 
LABORATORY MONITORING OF DTIS (PARENTERAL): ${ }^{5 B}$ Because there are no reversal agents for DTIs, elevated levels of these drugs carry the risk of life-threatening bleeding complications. ${ }^{6,7}$ To prevent overdosing and ensure adequate dosing, therapeutic drug monitoring is required for the majority of DTIs. ${ }^{7}$ Activated partial thromboplastin time (aPTT): If monitoring is indicated, all manufacturers of DTIs in the U.S. recommend using aPTT as the method of choice. The other indicators are prothrombin time (PT),activated clotting time(for renal impairment patients),thrombin time. As relatively new therapeutic agents, there is currently a lack of standardized laboratory tests for these anticoagulants. While the global clotting assays are widely available to monitor DTI therapy, they are not particularly specific, and the results have been shown to vary with reagents from different Manufacturers. ${ }^{7}$ As an alternative to clotting based tests, the chromogenic assays are specific, but currently lack widespread availability. ${ }^{8}$ Other indicators for laboratory monitoring of DTI are Activated clotting time (ACT), Ecarin clotting time(ECT), Prothrombin -induced clotting time (PiCT),Diluted thrombin time (Dtt).An increased focus has been placed on developing new laboratory tests suitable for the measurement of DTIs that are reproducible, accurate, and sensitive such as the ability to directly measure drug concentrations by mass spectrometry. $9,10,11$

ORAL ANTICOAGULANTS: Conventional drugs like coumarin derivatives: warfarin, Indanedione group: phenindione drugs and newer oral anticoagulants (DTI) are: Dabigatran and Rivaroxiban. They are indicated for prevention of venous thromboembolism in patients undergoing knee or replacement surgery.

Treatment in patients with atrial fibrillation or venous thromboembolism in recent decades has been based almost exclusively on the use of vitamin $K$ antagonists (warfarin). The use of old oral anticoagulants is always controversial because of the low therapeutic index; an excessive overdose is linked to the occurrence of hemorrhage. This situation requires continuous monitoring of INR (International Normalised Ratio) in the case of patients treated with oral coumarin anticoagulants like acenocoumarol or warfarin. So it is precise to repeated adjustments of doses that require analytical monitoring. For many years it has advocated the need to have more convenient new antithrombotic drugs. The developed new generation of antithrombotic is not related to coumarin derivatives.

The predictable pharmacokinetics and pharmacodynamics characteristics of Dabigatran and Rivaroxiban may facilitate management of patients who until now have been in treatment with traditional anticoagulants, given that it doesn't require routine laboratory monitoring in the vast majority of patients treated. They also present a profile of drug interactions very favourable. Among these Dabigatran is a direct thrombin inhibitor while Rivaroxiban is a factor $\mathrm{x}$ inhibitor (Rivaroxiban- is in clinical trial for treatment for DVT and prevention of atrial fibrillation) 
Fig No. 3: Mechanism of action of Dabigatran, Rivaroxiban and Warfarin

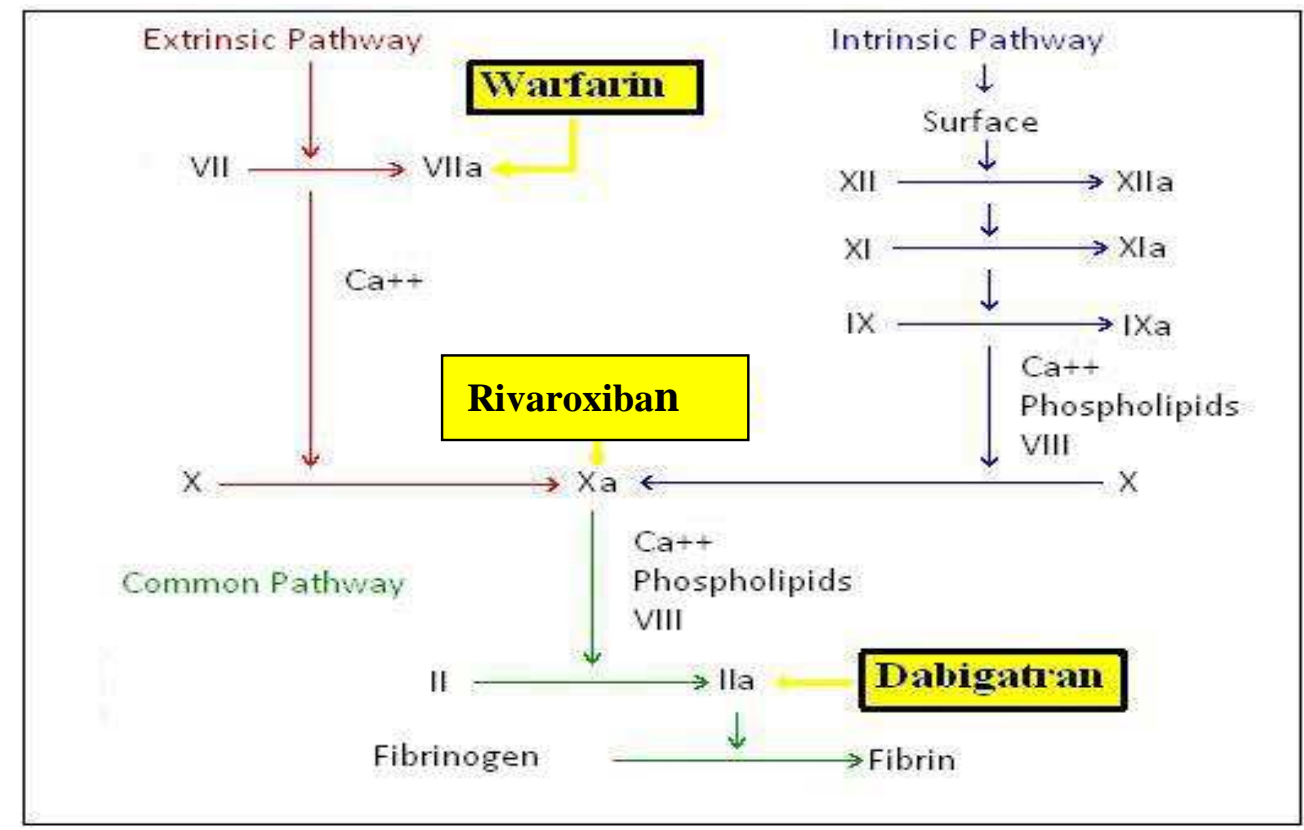

Table No 2: Comparison of different parameters of Dabigatran and rivaroxiban with warfarin

\begin{tabular}{|c|c|c|c|}
\hline Parameter & Warfarin & Dabigatran & Rivaroxiban \\
\hline Type & $\begin{array}{c}\text { Vitamin K- Dependent } \\
\text { Factors Inhibitor }\end{array}$ & $\begin{array}{c}\text { Direct Thrombin } \\
\text { Inhibitor }\end{array}$ & Direct Xa Inhibitor \\
\hline Half Life & 2.5 Days & $14-17$ hrs & 5- 13 Hours \\
\hline Elimination & $92 \%$ Renal & $\begin{array}{c}80 \% \text { renal \& } 20 \% \\
\text { biliary }\end{array}$ & $\begin{array}{c}33 \% \text { Renal (Unchanged) } \\
33 \% \text { Renal (As Inactive } \\
\text { Metabolites) } \\
33 \% \text { Biliary }\end{array}$ \\
\hline $\begin{array}{c}\text { Plasma protein } \\
\text { binding }\end{array}$ & $99.5 \%$ & $25-30 \%$ & $90 \%$ \\
\hline Bioavailability & $100 \%$ & Approximately $5 \%$ & $>80 \%$ \\
\hline
\end{tabular}

(i) Dabigatran: The maximum plasma concentration was reached between 30 minutes and 2 hours after oral administration, leading to a rapid onset of effect with a half life of 14 to 17 hours. Despite the low oral bioavailability, it shows low variability among individuals. The anticoagulant effect correlates well with plasma drug concentrations, which implies an effective anticoagulation with low risk of bleeding. In RELY trial by health Canada advisory dabigatrin (pradaxa) risk of major bleeds versus warfarin demonstrated significantly lower.11,12 Dabigatran is contraindicated in patients with severe renal impairment (creatinine clearance 30-50 ml/min) particularly in those at increased risk of bleeding. ${ }^{13}$ In pregnancy the medication should not be used unless the benefits outweigh the risks. It is not known if Dabigatran passes in to breast milk. Women taking this medication should not breast-feed. concomitant use of aspirin, 
clopidogrel, non-steroidal anti-inflammatory drugs anti-inflammatory drugs including COX-2 inhibitors (50\% more risk) ${ }^{14}$.

(ii) Dabigatran versus warfarin: The clinical effectiveness of dabigatran, which acts the end of the coagulation cascade, seems to be very different from the effect of warfarin, which acts almost at all levels of the cascade, despite having similar effects in terms of incidence of bleeding, treatment with Dabigatran did not require continuous monitoring and was not associated with the emergence of significant adverse effects.

(iii) Rivaroxiban: It is the first oral anticoagulant drug direct inhibitor of activated factor $\mathrm{X}$ of clotting. The inhibition disrupts the intrinsic and extrinsic pathways of coagulation cascade, there by the formation of thrombus is prevented. The purpose of this new oral anticoagulant (Rivaroxiban) is to replace vitamin $\mathrm{K}$ antagonists in the prevention of venous thromboembolism and arterial embolism in patients with atrial fibrillation. One of the most important advantages of Dabigatran and Rivaroxiban, compared to classical anticoagulants, is the lack of the need for continuous coagulation monitoring, which is much easier due to the stability of INR that is achieved with newer drugs. The highlight of newer oral anticoagulants is that they have fewer drug interactions because does not seem to inhibit any P450 enzyme. Dabigatran and Rivaroxiban does not require coagulation monitoring in the currently approved indications, however, there is no specific antidote, so that in case of overdose one should maintain adequate diuresis, haemostasis or the transfusion of fresh frozen plasma.

CONCLUSION: The emergence of new oral anticoagulants, such as Dabigatran and Rivaroxiban, has in recent years generated great expectations in the scientific community due to: the similar clinical effectiveness of warfarin, due to predictable pharmacokinetics, and pharmacodynamics and the adverse effects clinically less relevant. They have been found equivalent to LMWH in safety and efficacy. In future they may replace warfarin which has narrow therapeutic window, requires dosage adjustments and exhibits potential drug interactions.

\section{REFERENCES:}

1. Sharma HL, KK. Drugs Affecting Coagulation, Fibrinolysis and Platelet Functions. In Principles of Pharmacology, $2^{\text {nd }}$ edn .Hyderabad: Paras Medical Publishers; 2011.p.68182.

2. Tripathi KD. Drugs Affecting Coagulation, Bleeding and Thrombosis. In Essentials of

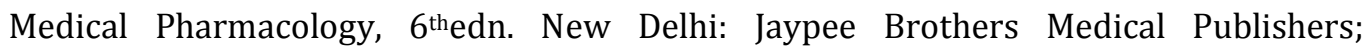
2010.p.603.

3. Sharma HL, KK. Drugs Affecting Coagulation, Fibrinolysis and Platelet Functions. In principles of Pharmacology, 2nd edn. Hyderabad:Paras Medical Publishers; 2011, p.6779.

4. Sharma HL, KK. Drugs Affecting coagulation, Fibrinolysis and Platelet Functions. In Principles of Pharmacology, $2^{\mathrm{n}}$ ed. Hyderabad: Paras Medical Publishers 2011, p:677.

5. Follis F, Filippone G, Montalbano G. Argatroban as a substitute of heparin during cardiopulmonary by pass:a safe alternative?Interact Cardiovasc Thorac Surg. 2010;10(4):592-6. 
6. Genzen JR, Fareed J, Hoppensteadt D. Prolonged elevation of plasma argatroban in a cardiac transplant patient with a suspected history of heparin-induced thrombocytopenia with thrombosis. Transfusion. 2010; 50(4):801-7.

7. Love JE, Ferrell C, Chandler WL. Monitoring direct thrombin inhibitors with a plasma diluted thrombin time. Thromb Haemost. 2007; 98(1):234-42.

8. Samama MM, Guinet C. Laboratory assessment of new anticoagulants. Clinical Chemistry and Laboratory Medicine. 2011;49(5):761-77

9. Molinaro RJ. Quantitation of argatroban in plasma using liquid chromatography electrospray tandem mass spectrometry (UPLC-ESI-MS/MS). Methods Mol Biol. 2010; 603;57-63.

10. Swan SK, Hursting MJ. The pharmacokinetics and pharmacodynamics of argatroban: effects of age, gender, and hepatic or renal dysfunction. Pharmacotherapy. 2000; 20(3):318-29.

11. Tran JQ, Di Cicco RA, Sheth SB, et al. Assessment of the potential pharmacokinetic and pharmacodynamic interactions between erythromycin and argatroban. J Clin Pharmacol. 1999;39(5): 513-19.

12. Eerenberg E.S, Kamphuisen P.w, Sijpkens M.K, Meijers J.c , Buller H.R, Levi M etal (2011). Reversal of Rivaroxaban and Dabigatran by Prothrombin Complex Concentrate: A Randomized, Placebo Controlled, Crossover Study in Health Subjects. Circulation 124(14):1573-9.

13. Van Ryn J, Stangier J, Haertter S, Liesenfeld K.H, Wienen W, Feuring M, Clemens A(2010).Dabigatran etexilate a novel reversible oral direct thrombin inhibitor :Interpretation of coagulation assays and reversal of anticoagulant activity .Thrombosis and haemostasis 103(6):1116-27.

14. Dabigatran:risk of serious haemorrhage-need for renal function testing. Drug safety update 5(5): A2.December 2011. 